\section{Restorative dentistry}

\section{Centric concerns}

Sir, we were saddened to read the description of the punishment meted out to some worn teeth in the second paper on Centric relation and increasing the occlusal vertical dimension. (BDJ 2021; 230: 83-89).

The first paper on the topic was riddled with old myths and fallacies about occlusion and wear, but the second paper caused us even more concern. It described a patient with localised anterior tooth surface loss with an obvious erosive component. All four canines and first premolars were in contact with one another and all the visible teeth had their marginal ridges intact - where most of their strength is. Traditional deprogramming devices are considered largely irrelevant when managing localised anterior tooth surface loss - unless wholly unnecessary gross dental destruction of moderately worn incisor teeth is chosen to be undertaken.

Sadly, six anterior teeth suffered an unprovoked attack with an airotor to provide one path of insertion for what Figure 22 described, euphemistically, as 'minimal reduction for 360-degree veneers'. However, that clinical picture showed complete removal of enamel to receive six ceramic full crowns which did about 40 years' worth of structural damage - apparently justified by some old nonsense about occlusion, vertical dimension and wear. ${ }^{1}$ That amount of elective, irreversible tooth destruction is not what we understand is normally involved when the word 'veneer' is used.

The biologically sensible approach to this sort of tooth wear was not further subtraction from the worn teeth, as described, but rather one utilising additive resin composite bonding, which was described as long ago as 2003 by Redman and colleagues ${ }^{2}$ and since then confirmed as being successful by many other clinicians in different locations. ${ }^{3,4,5,6}$

A systematic review by Mesko et al. ${ }^{7}$ concluded that 'rehabilitation with direct resin composite is undoubtedly more conservative than tooth preparations for full or partial indirect restorations [...] this offers good clinical results and satisfied patients'. Recent research has shown that when longevity of a restored anterior tooth is measured, crowns result in decreased longevity of the tooth when compared with direct restorations. ${ }^{8}$

Resin composite additions bonded at an increased anterior occlusal vertical dimension have been shown consistently to be very effective for managing worn teeth, while leaving all the strength inherent in their marginal ridges still available for the patient's future needs. Lastly, the supposed '360-degree veneers' term confounds us. We do like to use words properly and therefore ask gently 'did the consent record specify the word veneers?' If it did, then what was done to those unfortunate teeth might not be defensible on Montgomery consent grounds.

M. G. D. Kelleher, Bromley; F. J. T. Burke, Birmingham, UK

\section{References}

1. Kelleher M G D, Blum I B. Facts and fallacies about restorative philosophies for the management of the worn dentition. Prim Dent J 2020; 9: 27-31.

2. Redman C D J, Hemming K W, Good J A. The survival and clinical performance of resin-based composite restorations used to treat localised anterior tooth wear. BrDent J 2003; 194: 566-572.

3. Poyser N J, Briggs P FA, Chana H S, Kelleher M G D, Porter R W J, Patel M M. Evaluation of direct composite restorations for the worn mandibular anterior dentition - clinical performance and patient satisfaction. J Oral Rehabil 2007; 34: 361-376

4. Gulamali A B, Hemmings K W, Tredwin C J. Survival analysis of composite Dahl restorations provided to manage localised anterior tooth wear (ten year follow-up). Br Dent J 2011; 211: E9.

5. Kelleher M G D, Bomfim D I, Austin R S. Biologically based restorative management of tooth wear. Int J Dent 2012; doi.org/10.1155/2012/742509.

6. Milosevic A, Burnside $G$. The survival of direct composite restorations in the management of severe tooth wear including attrition and erosion: a prospective 8-year study. J Dent 2016; 44: 13-19.

7. Mesko M E, Sarkis-Onofre R, Cenci M S, Opdam N J, Loomans B, Pereira-Cenci T. Rehabilitation of severely worn teeth: A systematic review. J Dent 2016; 48: 9-15.

8. Burke F J T, Lucarotti P S K. The ultimate guide to restoration longevity in England and Wales: 9: incisor teeth: restoration time to next intervention and to extraction of the restored tooth. Br Dent J 2018; 225: 964-975.

https://doi.org/10.1038/s41415-021-2968-1

\section{Health and safety}

\section{'Never' fear}

Sir, it is pleasing to note that the Healthcare Safety Investigation Branch have removed extraction of the wrong tooth from the Never Event list due to 'strong and systemic safety barriers' not being in place. ${ }^{1}$ However, it is also good news in terms of training undergraduates. Fear of failure is a significant issue with the dental undergraduates and our future dental professionals. Indeed, the Good Childhood Report (2020) has reported that British teenagers have the lowest life satisfaction in Europe, in part due to fear of failure. ${ }^{2}$ As highlighted by the NHS Leadership Academy (2020) 'the word "never" can imply that someone has done something wrong and implies blame and liability. For staff it can lead to a feeling that they are to blame and can cause moral injury.' ${ }^{3}$

We all strive for the highest possible standards of patient care, and as such teach students to follow national and local guidance and welcome that any wrong site tooth extraction would still be considered by LocSSiPs. ${ }^{4}$ However, even the previous terminology and investigation processes created unintended consequences. Attempting to learn whilst in the heightened levels of anxiety caused by undertaking a potential Never Event procedure, can lead to avoidance behaviour and a reluctance to develop skills further. Therefore, the human factors involved in undertaking such procedures should be examined, and rather than Never Events, perhaps we should encourage a more positive culture to empower staff to ensure that following the relevant National Safety Standards are 'Always Events'.

K. Fox, Liverpool, UK

\section{References}

1. National Learning Report. Never Events: analysis of HSIB's national investigations. Available at: https:// www.hsib.org.uk/documents/272/HSIB_Never_ Events_-_analysis_of_HSIBs_national_investigations_ Report_V09.pdf (accessed March 2021).

2. The Children's Society. The Good Childhood Report 2020. Available at: https://www.childrenssociety. org.uk/information/professionals/resources/goodchildhood-report-2020 (accessed March 2021).

3. NHS Leadership Academy. Trauma and moral injury. 2020. Available at: https://people.nhs.uk/guides/ conversations-about-painful-subjects/steps/traumaand-moral-injury/ (accessed March 2021).

4. FGDP(UK), Royal College of Surgeons of England. LocSSIPs Toolkit Dental Extraction. Available at: https:// www.rcseng.ac.uk/dental-faculties/fds/publicationsguidelines/locssips-toolkit-dental-extraction/ (accessed 3 March 2021).

https://doi.org/10.1038/s41415-021-2969-0

\section{Orthodontics}

\section{Brackets response}

Sir, we thank the authors for their comments related to our letter ${ }^{2}$ on orthodontic brackets for dental trauma. We are not recommending the use of orthodontic brackets in the management of dental trauma without adequate training and/or supervision. Indeed, we wished to highlight the need to be suitably trained and confident to place them.

The Royal College of Surgeons of England guidelines state that clinicians may place bracket and wire splints if confident to do so. ${ }^{3}$ The British Society of Paediatric Dentistry guidelines on the management of traumatic dental injuries during the COVID-19 pandemic state that a skilled operator is needed when placing brackets and highlight the need for passive placement to avoid unwanted 